\title{
Ssams2, a Gene Encoding GATA Transcription Factor, Is Required for Appressoria Formation and Chromosome Segregation in Sclerotinia sclerotiorum
}

OPEN ACCESS

Edited by:

Hector Mora Montes,

Universidad de Guanajuato, Mexico

Reviewed by:

Birinchi Kumar Sarma,

Banaras Hindu University, India José Ascención Martínez-Álvarez, Universidad de Guanajuato, Mexico

*Correspondence: Hongyu Pan panhongyu@jlu.edu.cn

${ }^{\dagger}$ These authors have contributed equally to this work

Specialty section: This article was submitted to Fungi and Their Interactions,

a section of the journal

Frontiers in Microbiology

Received: 20 August 2018 Accepted: 23 November 2018 Published: 06 December 2018

Citation:

Liu L, Wang Q, Zhang X, Liu J, Zhang $Y$ and Pan H (2018) Ssams2, a Gene Encoding GATA Transcription

Factor, Is Required for Appressoria

Formation and Chromosome

Segregation in Sclerotinia sclerotiorum.

Front. Microbiol. 9:3031. doi: 10.3389/fmicb.2018.03031

\author{
Ling Liu', Qiaochu Wang', Xianghui Zhang, Jinliang Liu, Yanhua Zhang and \\ Hongyu Pan*
}

College of Plant Sciences, Jilin University, Changchun, China

AMS2, a multicopy suppressor for the cpn1 (SpCENP-A) mutant, functions to specifically regulate histone genes transcription and chromosome segregation. As a cell-cycle-regulated GATA transcription factor in eukaryotic organisms, little research has been done on the role of AMS2 protein in pathogenic fungi. In Sclerotinia sclerotiorum, Ssams2 (SS1G_03252) encodes a protein which has been predicted to contain GATA-box domain. Here, Ssams2-silenced strains with significantly reduced Ssams2 gene expression levels exhibited defect in hyphal growth, hyphal branching patterns, compound appressoria differentiation and the oxalic acid production compared to the wild-type (WT) strain. By common bean leaves infection assays, we identified the role of Ssams2 in full virulence. Furthermore, the numbers of cell nucleus in the same length of mycelium in Ssams2-silenced transformants were significantly less than that in the WT strain. The expression levels of histone genes and cell cycle genes in transformants were down-regulated significantly in the RNAi strains. Taken together, our work suggests that the TF SsAMS2 is required for growth, appressoria formation, virulence, and chromosome segregation in S. sclerotiorum.

Keywords: RNA interference, Sclerotinia sclerotiorum, SsAMS2, appressoria, virulence, chromosome segregation

\section{INTRODUCTION}

The Leotiomycetes fungus Sclerotinia sclerotiorum (Lib.) de Bary is a notorious plant pathogen, it infects over 600 plant species and incites rapid host tissue maceration in a non-discriminant manner (Liang and Rollins, 2018). Economical crops such as rape, soybean, sunflower, lettuce can be infected by S. sclerotiorum (Boland and Hall, 1994). As a host-nonspecific fungal pathogens, Sclerotinia diseases showed the limited tissue specificity and spreading necrosis (Rollins, 2003). S. sclerotiorum lacks of mitotic spores (Jurick Ii et al., 2004), but produces hardened, multicellular, highly melanized sclerotia which are capable survival for several years in hostile environment, including dry environments, low temperature, acidity, basicity, or microbial active soils (Bolton et al., 2006; Li et al., 2017). Sclerotia as a crucial structure in survivability and pathogenicity of S. sclerotiorum can differentiate either into vegetative hyphae or into apothecia which could release 
a lot of airborne ascospores to infect host plant and start a new disease cycle (Steadman, 1979; Wang et al., 2016; Fan et al., 2017).

S. sclerotiorum as a necrotrophic ascomycete can infect a broad range of plant species (Lecompte et al., 2013). To effectively infect plants the $S$. sclerotiorum have evolved sophisticated infection process (Kabbage et al., 2015). The compound appressorium (infection cushions) adhere to the host surface and penetrate the healthy plant cuticle (Uloth et al., 2016; Liang and Rollins, 2018). Infection cushions were the multicellular, melanin-rich hyphal penetration structure (Li et al., 2017). When contact with host, the hyphae of $S$. sclerotiorum become extensively branched, exhibit hook and bifurcate (Lumsden and Dow, 1973; Tariq and Jeffries, 1984; Jamaux et al., 2007; Huang et al., 2008; Xiao et al., 2014). Sticky mucilage around the infection cushions increase the adhesion of S. sclerotiorum hyphae (Tariq and Jeffries, 1984), and thin penetration pegs originate from compound appressorium can perforate plant cuticle (Huang et al., 2008; Xiao et al., 2014). Oxalic acid (OA) as a non-hostselective toxin, play very important roles in phytopathogenic fungi (Liang et al., 2015a; Xu et al., 2015). In S. sclerotiorum, $\mathrm{OA}$ is released and macerated the host tissues, help the pathogen kill the host (Andrew et al., 2012; Heller and Witt-Geiges, 2013). Therefore, if the hyphae growth, OA production or the appressorium differentiation were blocked, Sclerotinia diseases could be controlled effectively.

GATA type zinc finger transcription factor (TF) was widely found in eukaryotes, plays critical roles in regulating physiological metabolism and controlling multicellular development (Lowry and Atchley, 2000; Liu et al., 2018). The ams 2 gene, a multicopy suppressor for the cpn1 mutant in fission yeast (Chen et al., 2003), encodes a GATA factor, binds to the histone gene promoter regions and takes part in transcriptional activation of the histone genes (Takayama et al., 2016). In Schizosaccharomyces pombe, AMS2, assembled in promoter regions of putative target genes and enriched in central centromeres, is critical for chromosome segregation, possibly via regulating localization of SpCENP-A ( $c p n 1)$ which is the centromere-specific histone $\mathrm{H} 3$ variant (Takayama et al., 2008). In $S$ phase of cell cycle, an amount of histone is synthesized and appropriate histone gene expression levels are essential for chromosome segregation and genome integrity (Gunjan et al., 2005). The expression of ams 2 comes to the peak in $\mathrm{S}$ phase and the deletion or overproduction of ams 2 affects the normal synthesis of histones (Takayama et al., 2010). In ams2-deficient cell, the division cycle of the mutant cell is longer and the histone genes accumulation were markedly reduced during DNA replication (Takayama and Takahashi, 2007). Overproduction of AMS2 affect the normal meiosis process, histone deposition into centromeres and genetic stability (Trickey et al., 2013). The cell cycle relevant ams 2 expression level is important to the centromere nucleosome formation and genetic stability.

Because AMS2 protein is vital to chromosome segregation and play a role in the cell division, we closely examine the SsAMS2 protein as a $S$. pombe AMS2 homolog in S. sclerotiorum. We probed the $S$. sclerotiorum genome and used RNAi strategy to down-regulate the Ssams2 gene expression. The results showed that SsAMS2 protein was involved in hyphal growth, appressoria formation, and chromosome segregation.

\section{RESULTS}

\section{Identification and Sequence Analysis of Ssams2}

From the genome of $S$. sclerotiorum, nine proteins containing GATA-domains are predicted based on PFAM (Amselem et al., 2011; Liu et al., 2018). Among them, a gene (SS1G_03252) which encode a protein contains 1201 amino acids. Phylogenetic analysis demonstrate that SsAMS2 homologous proteins can be found in Homo sapiens, Danio rerio, Drosophila melanogaster, Mus musculus, and S. pombe (Figure 1A). Sequence alignment of SsAMS2 was performed and phylogenetic analysis using different fungi species, such as Marssonina coronariae, Metarhizium brunneum, Botrytis cinerea, Metarhizium majus, Colletotrichum fioriniae, Aspergillus flavus, Aspergillus bombycis, Talaromyces marneffei, and S. pombe. The results displayed that GATA boxcontaining protein (SS1G_03252) is classed with the AMS2 TF (Figures 1A,B). Taken together the results from the phylogenetic analysis, the choosed protein in S. sclerotiorum was named as SsAMS2. To examine the expression patterns of Ssams2 during hyphae, sclerotial and apothecium developmental stages in S. sclerotiorum, qRT-PCR was used to test the expression levels of Ssams2. The results indicate that the Ssams2 was downregulated expression during the sclerotia and apothecium stages (Supplementary Figure S1).

\section{RNAi-Mediated Down-Regulation of Ssams2 Impairs Mycelium Growth}

To determine whether SsAMS2 influences the mycelium development of $S$. sclerotiorum, the Ssams2 knockdown transformants were obtained. S. sclerotiorum is a multi-nucleated fungus and RNA silencing is a mature gene function research strategy in fungi (Lyu et al., 2016; Liu et al., 2018). The result of sequence alignment (Blastn) showed that the Ssams2 gene sequence was highly specific (Supplementary Figure S2). Two Ssams2 target fragments which have no overlapping and outside of GATA zinc finger domain were designed (Figure 2A). The Target 1 (254 bp) and Target 2 (352 bp) fragments were ligated into the pSilent-Dual 1 (pSD1) vector (Nguyen et al., 2008; Fan et al., 2017; Liu et al., 2018; Figure 2B). The resulting constructs (Figures $2 \mathbf{A}, \mathbf{B}$ ) were used to transform the S. sclerotiorum protoplast (Rollins, 2003; Qu et al., 2014). The transformants were selected on PDA medium with geneticin and confirmed through the amplification of the geneticin resistance gene (Supplementary Figure S3). Six transformants were designated as Ssams2-T1-93, Ssams2-T1-98, Ssams2-T1-17 (originated from Target 1 transformation) and Ssams2-T2-202, Ssams2-T2-102, Ssams2-T2-108 (originated from Target 2 transformation). The Ssams 2 transformed strains obtained from the two targets showed a similar phenotype and we can trust the accuracy of the RNAi strategy (Lyu et al., 2015; Liu et al., 2018). 
A

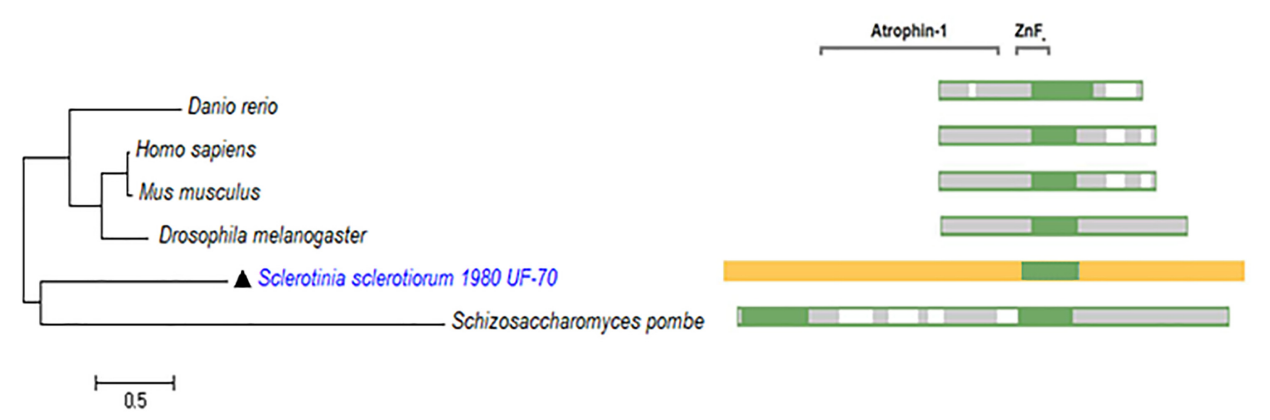

B

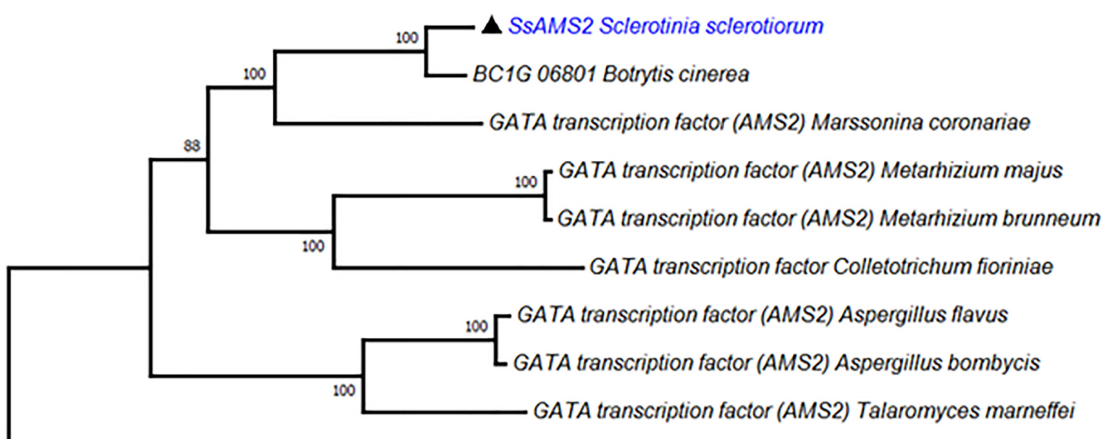

GATA-type transcription factor AMS2 Schizosaccharomyces pombe

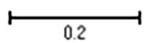

FIGURE 1 | SsAMS2 is a GATA-TF and highly conserved among fungi. (A) SsAMS2 sequence was used to SmartBLAST with NCBI, the query sequences by Danio rerio, Mus musculus, Homo sapiens, Drosophila melanogaster, and Schizosaccharomyces pombe were choosed, white color represents the gaps, green color represents landmark matches. (B) Phylogenetic analysis of S. sclerotiorum SsAMS2 and other homologous GATA transcription factors from Botrytis cinerea, Marssonina coronariae, Metarhizium brunneum, Metarhizium majus, Colletotrichum fioriniae, Aspergillus flavus, Aspergillus bombycis, Talaromyces marneffei, Schizosaccharomyces pombe, SsAMS2 marked with $\mathbf{\Delta}$.

SsAMS2 is homologous to protein AMS2, which has been demonstrated to be involved in activation the core histone genes expression at $\mathrm{S}$ phase of cell cycle in $S$. pombe (Takayama et al., 2016). In ams2-deficient cell, the division cycle is longer than that in wild-type cells (Takayama and Takahashi, 2007). Therefore, we hypothesized that SsAMS2 was involved in hyphal development in S. sclerotiorum. The RNAi transformants exhibited defect in mycelial growth. Both the Target 1 transformants and the Target 2 transformants exhibited impaired growth phenotypes (Figures 2C,D). The mycelium colony diameter of RNAi strains was significantly lower than that of WT, Ssams2 gene was down-regulate expression and the cell growth rate was reduced (Figure 2E). qRT-PCR was used to verify the expression of Ssams 2 in different strains. The results showed that the accumulation of Ssams2 gene at mRNA level were suppressed in different degrees (Figure 2F). Taken together, we found the similar phenotype within the Target 1 and the Target 2 transformants, so the Target 1 transformants were choosed for the subsequential experiments. The hyphal micro-morphology was observed under light microscope and found the hyphal branching patterns of Ssams2-silenced strains were perpendicular and short, whereas the wild-type strains were acute angle branching (Figure 3A). The results showed that Ssams2 is associated with hyphal growth and hyphal branching patterns in S. sclerotiorum.
The RNAi strains demonstrate abnormal sclerotial development. With the change of mycelium growth morphology, the number of sclerotia decreased and the distribution was different (Figure 2D and Supplementary Figure S4).

\section{Ssams2 Is Involved in the Virulence of S. sclerotiorum}

We have showed that reduced the expression of Ssams2 could impaired the hyphal growth and hyphal branching patterns (Figures 2, 3A). In order to verify that Ssams2 take part in the formation and development of compound appressoria (infection cushions), the experiment to induce the generation of compound appressoria was completed. In this assay, Ssams2-silenced transformants failed to produce infection cushions on glass slide. While, the WT strain had already produced mature infection cushion structures once contact with glass slide (Figure 3B). Mycelial plugs were inoculated on PDA medium covered with cellophane and microscopic observation revealed that the hyphal tips of RNAi transformants exhibited hook and bifurcate which like the initiation of compound appressorium. However, the hook and bifurcate did not develop into multihyphal appressoria or infection cushions (Figure 3C). WT, Ssams2silenced strains and a mutant strain which affect hyphal growth 

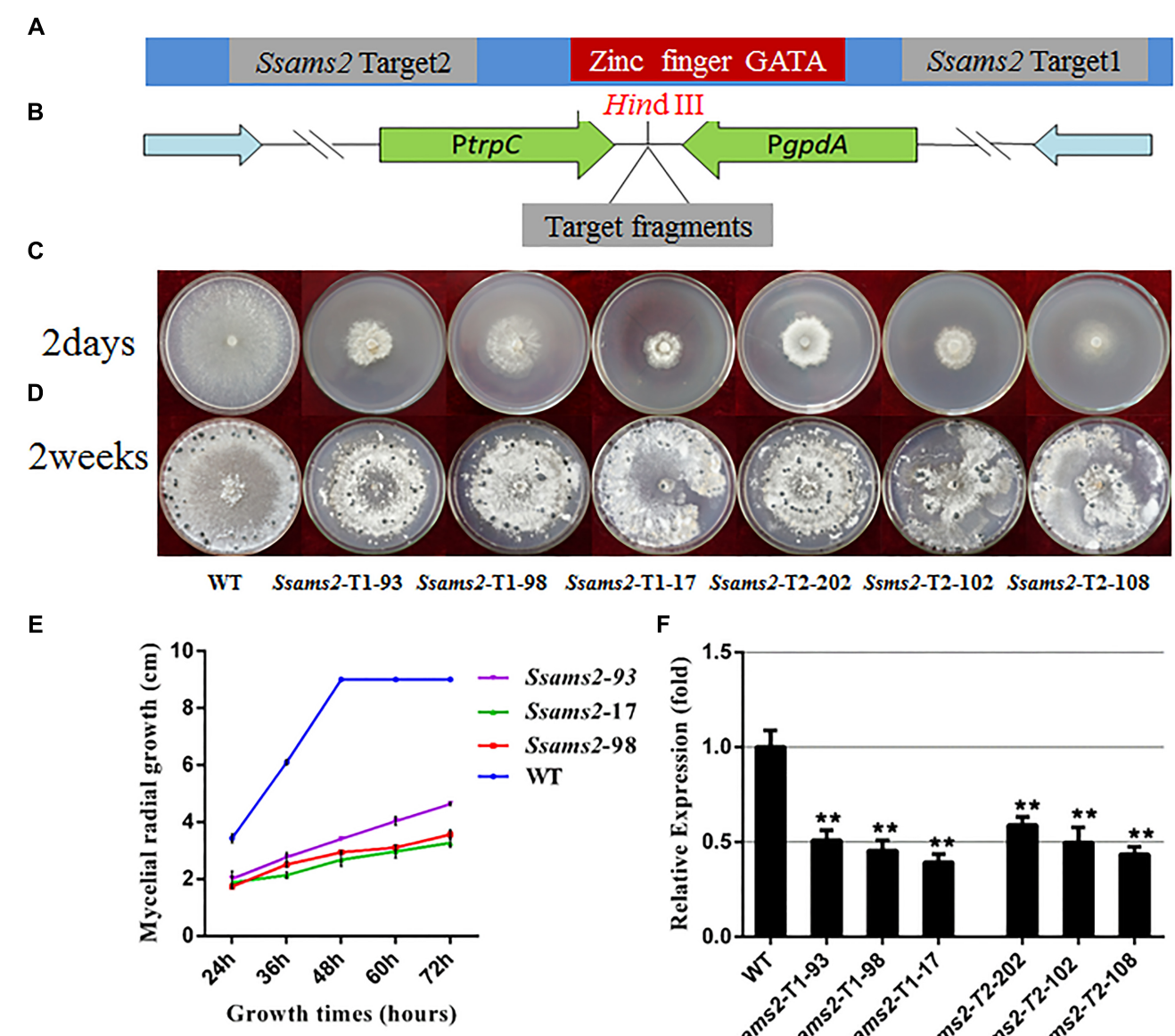

WT Ssams2-T1-93 Ssams2-T1-98 Ssams2-T1-17 Ssams2-T2-202 Ssms2-T2-102 Ssams2-T2-108

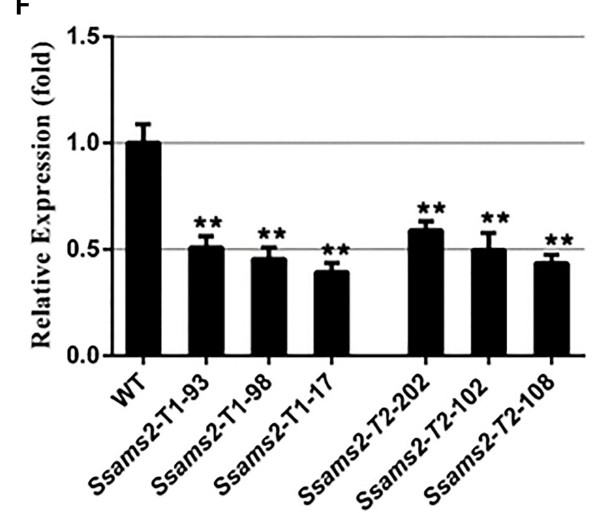

FIGURE 2 | Morphologic observation of the Ssams2-silenced strains. (A,B) The silence vectors construction of Ssams2 gene in S. sclerotiorum. (C) Mycelium growth of WT, Ssams2-Target 1-silenced strains and Ssams2-Target 2-silenced strains. (D) Sclerotial morphology after 2 weeks of growth. (E) Hyphal growth rates of the RNAi strains and the WT strain cultured on PDA plates. (F) The Ssams2 gene expression level was verified by qRT-PCR (one-way ANOVA, **indicates significance at $p<0.01)$.

but does not affect oxalic acid secretion (obtained and kept in our lab) were inoculated on PDA medium supplemented with bromophenol blue (BPB). The result confirmed that Ssams2-silenced transformants showed the OA-minus phenotype (Figure 3D and Supplementary Figure S4).

To examine how SsAMS2 is involved in process of pathogenic in $S$. sclerotiorum, the detached common bean leaves were inoculated with the agar plugs derived from WT strain and Ssams2-silenced transformants. The results show that the RNAi strains infection capacity were highly impaired in the infection process (Figures $\mathbf{4 A}, \mathbf{C}$ ). The observation that genetic mutants defective in compound appressorium formation exhibit virulence defects that can be rescued by wounding the host (Li et al., 2012). To monitor the penetration rescued events, host tissues were wounded then inoculated with the agar plugs and placed to the same condition as to the healthy leaves. RNAi strains distinct primary lesions emerged later than the WT strain. The area of the wounded host leaf colonized by RNAi strains was smaller than WT strain but the bigger necrotic lesions compared to the unwounded leaf (Figures 4B,D). These results suggested that virulence defects that can be partial rescued by wounding the host, the absence of Ssams 2 affect the production of compound appressorium and reduced penetration rates in S. sclerotiorum.

\section{Chromosome Segregation Is Disturbed in Ssams2-Silenced Strains}

In fission yeast, overexpression of ams 2 could suppress the chromosome missegregation phenotype (Lim et al., 2015). To verify the role of Ssams2 in the chromosome segregation phenotype, 4',6-diamidino-2-phenylindole (DAPI) dye was used for routine nuclear staining, cell nucleus in hyphae and germinating protoplasts (PDA) were observed under fluorescence microscope (Figure 5). In the wild type hyphae, cell nuclei were intensive and overlap, but in Ssams2-silenced transformants the cell nuclei were more dispersed. Both in hyphae and in germinating protoplasts, the numbers of cell nucleus in the same length of mycelium in Ssams2-silenced transformants were significantly less than that in the WT strain 


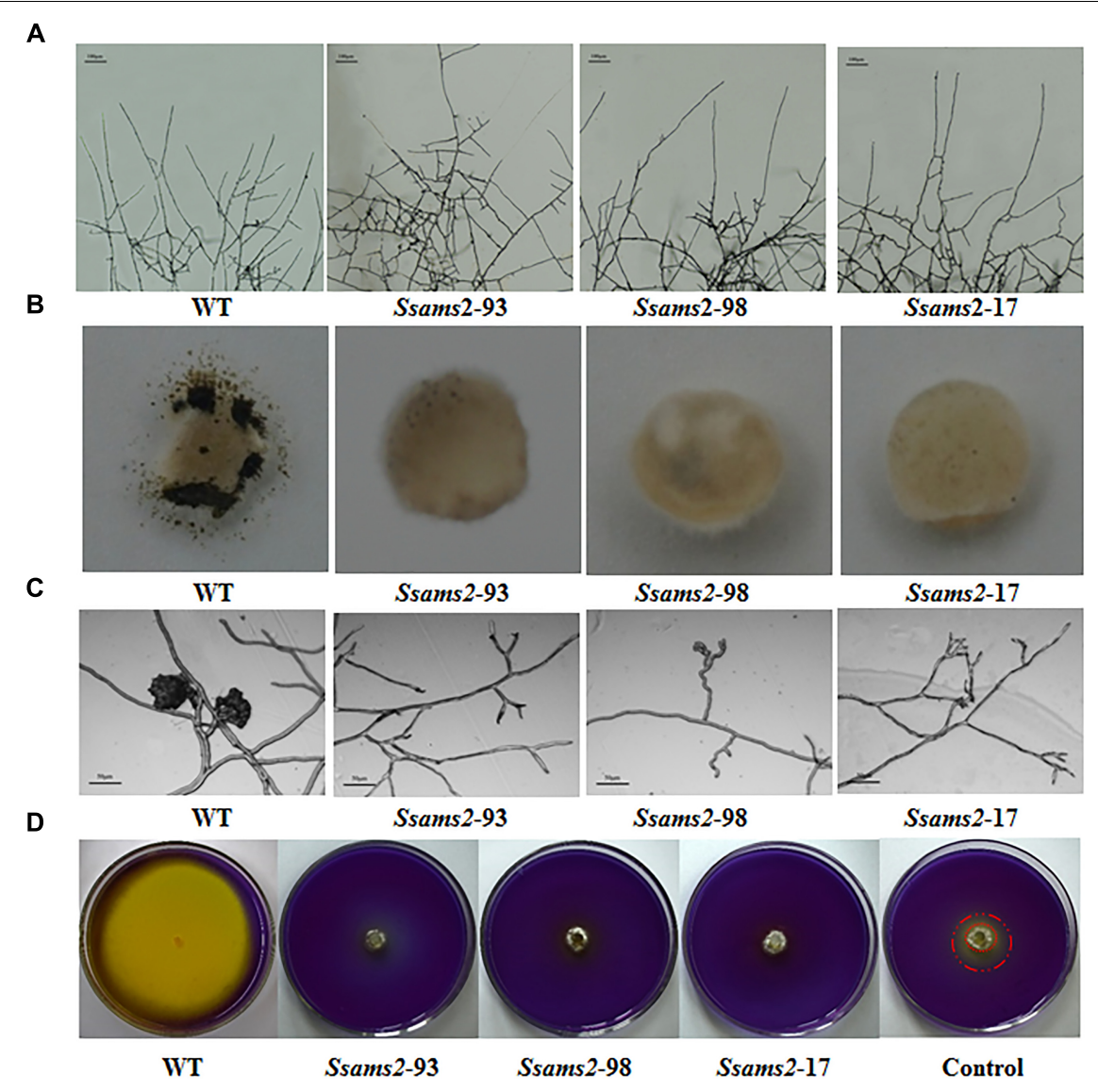

FIGURE 3 | Infection cushions development and OA production assayed. (A) The observation of mycelium branches from the RNAi strains and the WT strain. The photographs were taken after $16 \mathrm{~h}$ with a light microscope. Scale bars represent $100 \mu \mathrm{m}$. (B) Infection cushions production of WT strain and Ssams2-silenced strains. Mycelia plugs were inoculating on glass slid and the pictures were obtained after $3 \mathrm{dpi}$ (5 mm in diameter). (C) Infection cushions on cellophane overlaid PDA were observed by light microscopy 16 hpi. Scale bars represent $50 \mu \mathrm{m}$. (D) WT and Ssams2-silenced strains were assayed for OA production on PDA medium supplemented with bromophenol blue. The control was other mutant which mycelium grows slowly but does not affect oxalic acid production.

(Figure 6). The results demonstrated that Ssams2 is required for chromosome segregation in S. sclerotiorum.

\section{Ssams2 Is Involved in Histone Genes and Cell Cycle Genes Expression}

We have showed that reduced the expression of Ssams 2 could impaired chromosome segregation. Appropriate levels of histone expression are critical for DNA replication and chromosome segregation (Gunjan et al., 2005). ams2 of $S$. pombe which is homologous with Ssams 2 has been demonstrated to be essential for histone genes transcription (Takayama and Takahashi, 2007). The histones (H2A, H2B, H3, H4, CENP-A in S. sclerotiorum), cell cycle related proteins (CDC6 and CDC28 in S. sclerotiorum) and SsAMS2 were analyzed using STRING database ${ }^{1}$ to derive the protein-protein interaction (PPI) network (Hsia et al., 2015) and the potential signaling pathways, SsAMS2 is directly related to SsCDC6 and SsCENP-A (Figure 7A). The expression levels of the cell cycle related gene Sscdc6 (SS1G_04516), Sscdc28 (SS1G_02296), histone genes H2A (SS1G_10959), H2B

\footnotetext{
${ }^{1}$ https://string-db.org/
}

(SS1G_10960), H3 (SS1G_09608), H4 (SS1G_09609), and Sscpn1 (SS1G_00304) in the RNAi transformants were down-regulated significantly (Figures $\mathbf{7 B}-\mathbf{H}$ ). These data demonstrate that chromosome segregation was disturbed in Ssams2-silenced strains, the transcription level of histone genes and cell cycle genes also were impaired.

\section{DISCUSSION}

In $S$. pombe, AMS2 as GATA transcriptional factor (TF) could activate histone genes transcription. After overproduced AMS2, chromosome instability and all the core histone genes were augmented expression (Takayama et al., 2010). The ams 2 gene is required for centromere function (Chen et al., 2003), destruction of ams 2 can help to prevent core histone genes expression. AMS2 stability expression is also required to ensure the coupling of mitosis to DNA replication (Trickey et al., 2013). So, whatever the AMS2 overproduction or absence, the defects in global cell division, histone genes expression, central centromere and chromosome stability can occur 

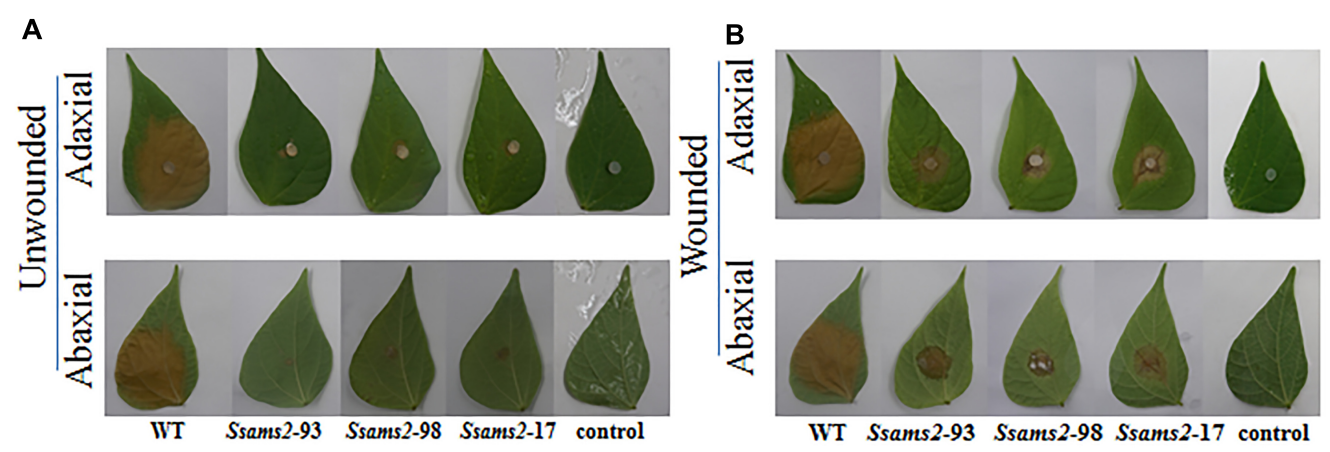

C

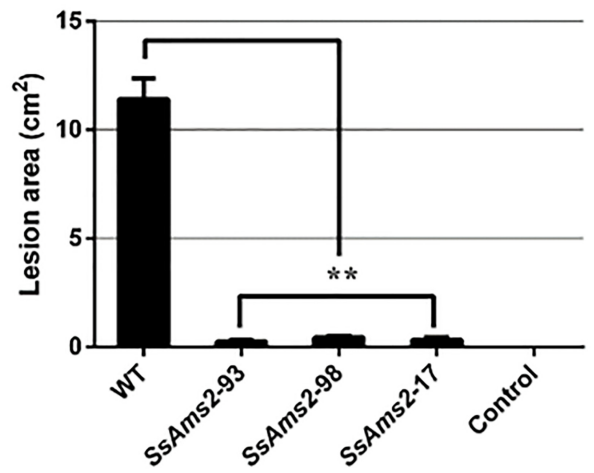

D

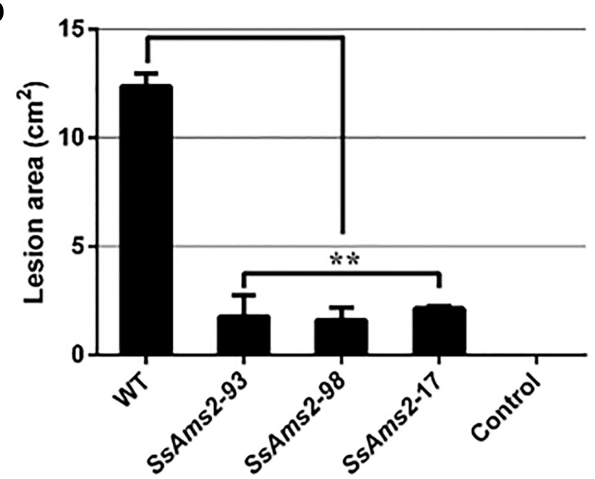

FIGURE 4 | The absence of Ssams2 gene affects full virulence of S. sclerotiorum. (A,B) Ssams2-silenced strains produced relatively small spots on the host leaves at 36 hpi (A: unwounded leaves; B: wounded leaves). The control was medium plug without fungi. The experiment was repeated three times. (C,D) The statistic analysis of the lesion size in panels $(\mathbf{A}, \mathbf{B})$. (**indicates significance at $p<0.01$ ).

(Takayama and Takahashi, 2007; Takayama et al., 2010). In ams 2 mutant cells, the total amount of SpCENP-A deposition in the central centromeres was decreased compared to in the wild-type controls and showed missegregation of chromosome (Takahashi et al., 2005). The colony formation of fission yeast was exceedingly slow after the ams 2 gene was deleted and displayed abnormal mitosis accompany the aberrant chromosomes segregation (Chen et al., 2003). GATA TFs are broadly distributed in eukaryotes, fungal GATA factors have been demonstrate to be associate with diverse functions such as nutrient signal response, mating type switch, nitrogen control, siderophore biosynthesis, light regulated photomorphogenesis, circadian regulation, developmental differentiation, and morphogenesis (Tsang et al., 1997; Perlman et al., 1998; Charron et al., 1999; Reyes et al., 2004). In this study, SsAMS2 as a GATAbox contains protein homologous to the AMS2 in S. pombe (Figure 1). Based on the roles of AMS2 orthologs, we speculated that SsAMS2 may be involved in fungal growth, development and chromosome segregation in S. sclerotiorum. As a method to reduce the transcript accumulation, RNAi strategy was used to investigate the function of Ssams2. Compared with the wild-type strain, the Ssams2-silenced strains slowed hyphal growth and development, produced hyphae with wider angled branches and damaged the formation of infection cushion (compound appressoria) (Figures 2, 3).

Sclerotinia sclerotiorum, an aggressive phytopathogen, infects hundreds of species of plant and cause huge economic damage
(Guyon et al., 2014; Yang et al., 2018). As a host-nonspecific fungus, the $S$. sclerotiorum infection process has evolved more sophisticated and comprehensive (Lyu et al., 2016; Liu et al., 2018). Recently, a new infection mechanism, two-phase compatibility model by $S$. sclerotiorum was proposed. At first stage, the pathogen avoids host basal defense reactions and evades recognition. During this stage, the compound appressoria can penetrate cuticle but cannot penetrate the plant cell walls, the pathogen involves a transient biotrophic interaction stage (Kabbage et al., 2015; Liang and Rollins, 2018). Following this stage, mycelium which grows within the apoplastic space secrete $\mathrm{OA}$, cell wall degrading enzymes and other pathogenicity factors, kill the plant cells and the fungus switch to necrotrophic growth (Kabbage et al., 2015; Liang and Rollins, 2018).

Compound appressoria are hyphal tip-differentiated multicellular infection structures formed by many plantpathogenic fungi on the host surface (Hofman and Jongebloed, 1988; Boenisch and Schäfer, 2011). In S. sclerotiorum, impenetrable surfaces, such as dialysis tubing, parafilm, plastics, glass, and cellophane are often efficiently triggering compound appressoria differentiation (Li et al., 2018). Compound appressoria is critical for penetrating into the host cells and the complexity of compound appressorium is relevant to physical resistance to penetration on the host surface (Tariq and Jeffries, 1984; Jamaux et al., 2007; Huang et al., 2008). The penetration pegs breach the cuticle layer, bulbous and multi-lobed subcuticular vesicles form and subcuticular 


\section{A}

B
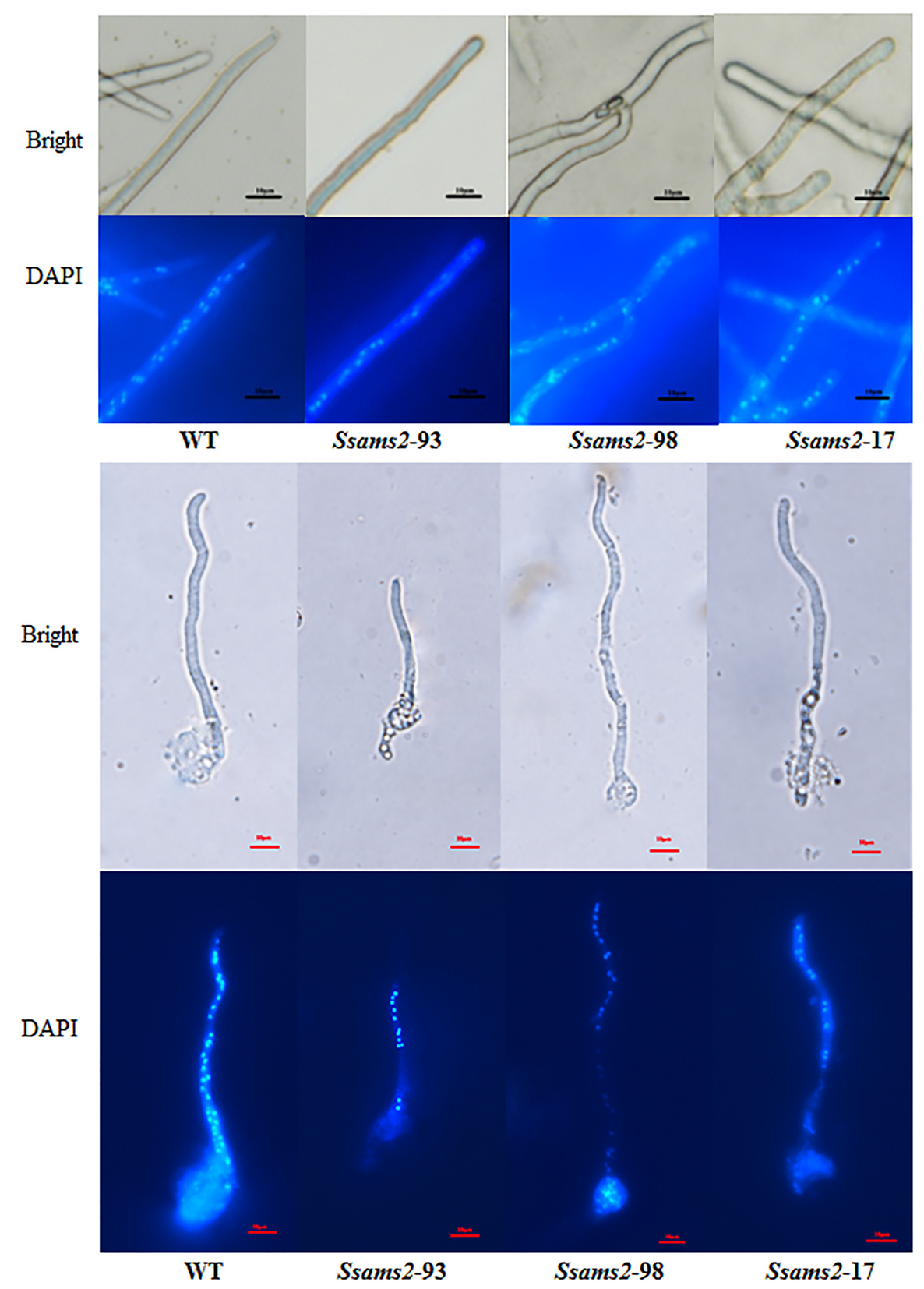

FIGURE 5 | Cell nucleus observation in hyphae of Ssams2-silenced transformants and WT strain. Hyphae (A) and the germinating protoplasts (B) were stained by 4',6-diamidino-2-phenylindole (DAPI) in the dark for $30 \mathrm{~min}$ and observed in fluorescence microscope. Scale bar represent $10 \mu \mathrm{m}$.

infection hyphae were produced which spread horizontally beneath the cuticle to comprise the leading colonization front (Li et al., 2012; Liang and Rollins, 2018). The deletion of the genes associated with compound appressoria formation leads to defect in appressorium formation and infection initiates (Liang et al., 2015b). For example, Sscaf1 (compound appressorium formation related gene 1), which encodes a secretory protein and is required to infection cushions formation, plays a key role in host penetration (Xiao et al., 2014). Likewise, Ssggt1 ( $\gamma$-glutamyl transpeptidase) regulate substrates GSH/GSSG balance, and function in compound appressorium development, Ssggt1 mutants revealed a decrease in infection efficiency ( $\mathrm{Li}$ et al., 2012). In this study, WT strain rapidly differentiated infection cushions from vegetative hyphae on contact with glass slide. However, very few infection cushions were differentiated by the Ssams2-silenced strains (Figure 3B). The RNAi and WT strains were inoculated on common bean leaves, the Ssams2-silenced strains showed significant virulence defects (Figure 4).

Additionally, OA as a virulence factor has been demonstrated by studies, the full virulence requires a dynamic control of 


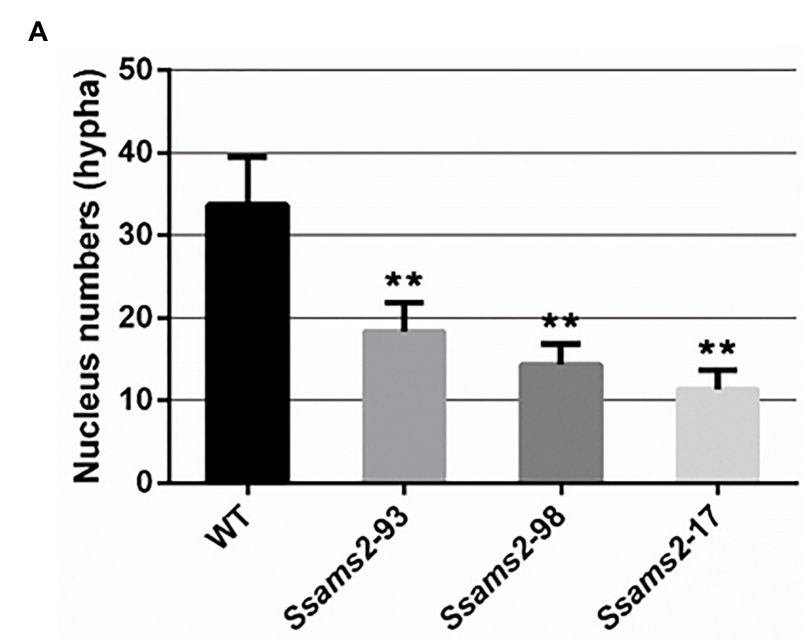

B

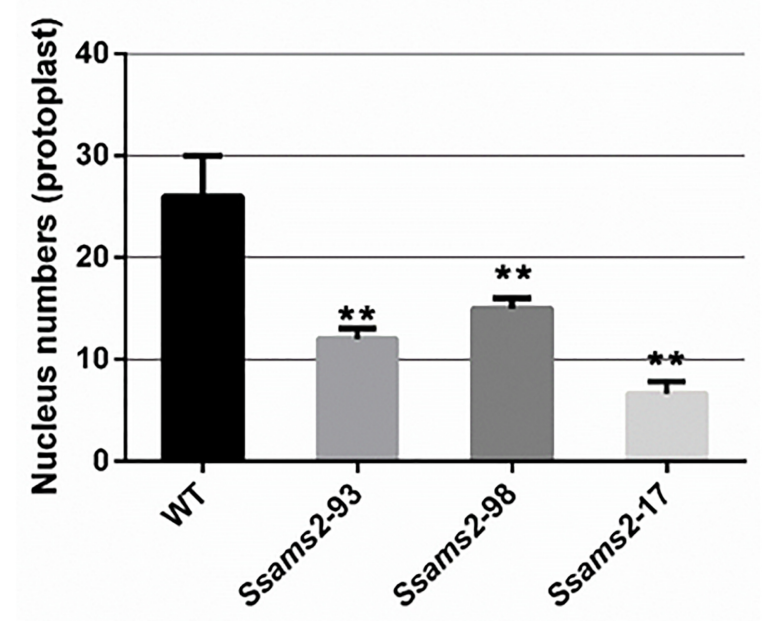

FIGURE 6 | The numbers of cell nucleus were counted in hyphae of Ssams2-silenced transformants and WT strains. Count the numbers of cell nucleus in the same length of mycelium (A) and germinating protoplast (B) Values represent the means \pm standard deviation (SD) $(n=3)$. Asterisks indicate significant difference compared with the WT strain $\left[{ }^{* *} p\right.$-value $<0.01$, one-way analysis of variance (ANOVA)].

OA accumulation through multiple mechanisms (Liang and Rollins, 2018). OA accumulation lowers the ambient $\mathrm{pH}$ and the production of $\mathrm{OA}$ is critical for host colonization (Cessna et al., 2000; Rollins and Dickman, 2001; Davidson et al., 2016). Sshs 1-silenced strains showed a defect in compound appressoria formation, OA secreted decreased and virulence defect (Yu et al., 2017). The Ssnox1 mutant exhibited reduced efficiency in oxalate production, attenuated virulence (Kim et al., 2011). In this study, previous results show that Ssams2 play an important role in hyphae growth, we hypothesize that the slowed mycelium growth caused the defect virulence of RNAi strains. The assayed of OA production on PDA supplemented with $\mathrm{BPB}$ showed that the less OA secreted could not solely attribute to the slowed mycelium growth (Figure 3D). The secreted levels of OA in Ssams2-silenced strains were lower than that in WT strain (Figure 3D and Supplementary Figure S5). The leaves which wounded prior to infection was used, showed partially rescue in infection efficiency compared with the healthy host. From the above, in $S$. sclerotiorum, the weaken of virulence might be associated with the growth and development of mycelium, compound appressoria formation, and OA secreted rather than a single factor. These results demonstrate that Ssams2 involved in hyphae growth, compound appressorium formation and OA secreted, these factors common leading to failure in host penetration, attenuated virulence.

In eukaryotic cells, the two of each of the $\mathrm{H} 2 \mathrm{~A}, \mathrm{H} 2 \mathrm{~B}, \mathrm{H} 3$, and $\mathrm{H} 4$ comprise the nucleosome which is the basic unit of chromatin (Zhou et al., 2015; Takayama et al., 2016). The core histone genes showed evolutionarily conserved organization and appropriate transcription levels are essential for DNA damages repaired, chromosome segregation, mitosis, spermatogenesis, and cell viability (Osley, 1991; Maruyama et al., 2006; Marzluff, 2010; Takayama et al., 2016; Franciosi et al., 2017). AMS2 binds to the AACCT-box which exist in all core histone gene promoters via the zinc finger motif (Takayama et al., 2016), which is critical for activating histone genes transcription with Teb1 in cell cycle S phrase (Valente et al., 2013). Cell cycle-dependent ams2 levels fluctuating manner is crucial for the centromere nucleosome formation and genetic stability (Takayama et al., 2010; Trickey et al., 2013). In the ams 2 mutants, the cell cycle was disturbed and the process of transcriptional oscillation of histone genes was disappeared (Takahashi et al., 2005). On the other hands, accompany DNA replication, the cellcycle-dependent accumulation of histone mRNAs was markedly suppressed (Takayama and Takahashi, 2007). In both yeast and higher eukaryotes, the DNA replication was interfered with by genotoxic agents which impaired histone gene expression (Han et al., 1987; Nelson et al., 2002; Gunjan et al., 2005). In yeast, a mutant form of CDC6 that lacked its enzyme activity was unable to copy their DNA and the cell did not divide (Chang et al., 2015). Kinetochore is indispensable for chromosome segregation and CENP-A as a centromere-specific histone $\mathrm{H} 3$ variant plays an important role for the formation of kinetochore (Chen et al., 2003). AMS2 GATA factor which promotes histone gene activation is required for CENP-A deposition (Takayama et al., 2008). PPI network obtained from STRING database showed that SsAMS2 interacts with SsCDC6 and SsCENP-A directly, implicating that SsAMS2 might involve in DNA replication and chromosome segregation in S. sclerotiorum. In this study, we found that the numbers of cell nucleus in Ssams2-silenced transformants were significantly reduced (Figure 6). It is likely that the less expression of Sscnp1, Sscdc6 and histone genes is due to the reduced transcription accumulation of $S s a m s 2$ by RNAi strategy (Figure 7). It is well defined that proper Ssams2, Sscnp1 and core histone genes accumulation are vital for normal DNA replication and chromosome segregation.

In conclusion, the Ssams 2 gene is characterized to encode a GATA-type transcription factor orthologous to the $S$. pombe ams 2 gene which is required for efficient core histone genes and cell cycle genes transcription. This study provides evidence that Ssams2 plays a critical role in the histone genes transcription 
A

C
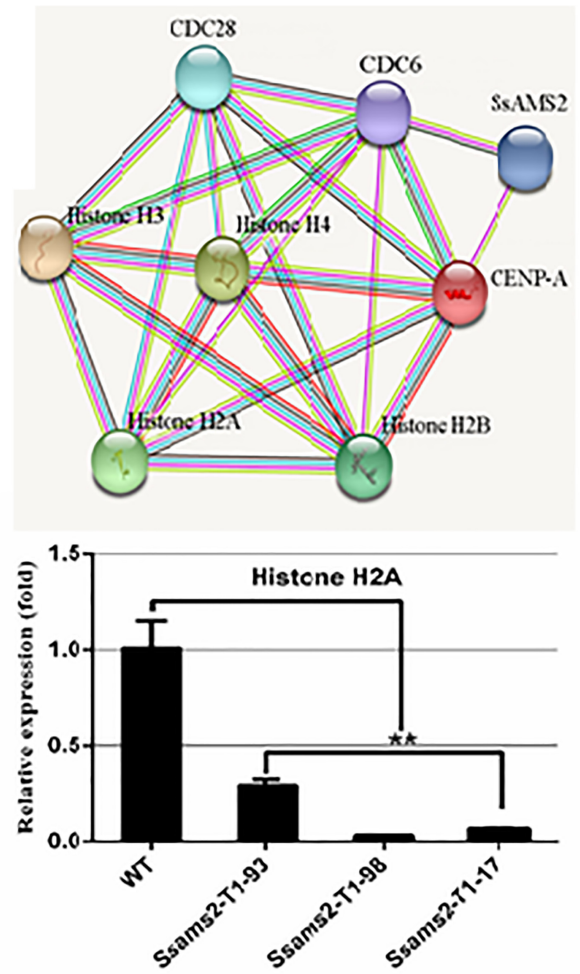

E

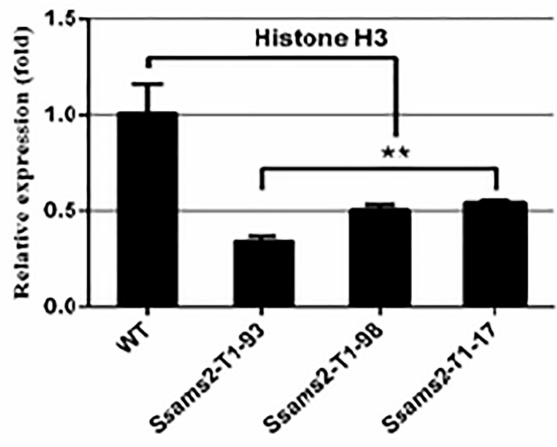

G

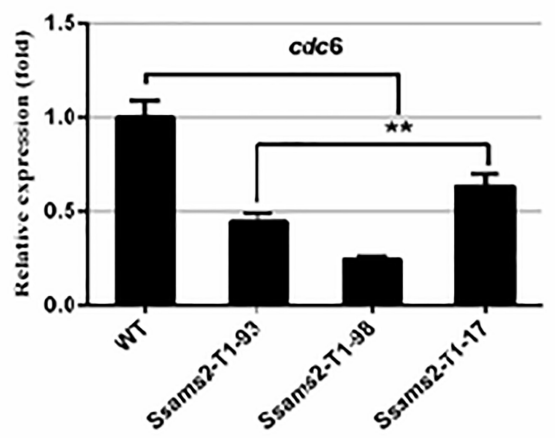

B

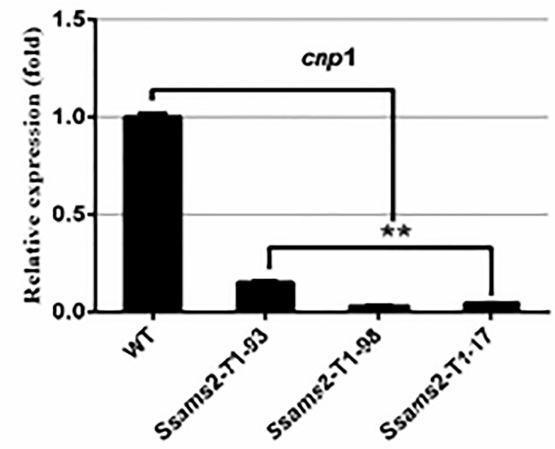

D

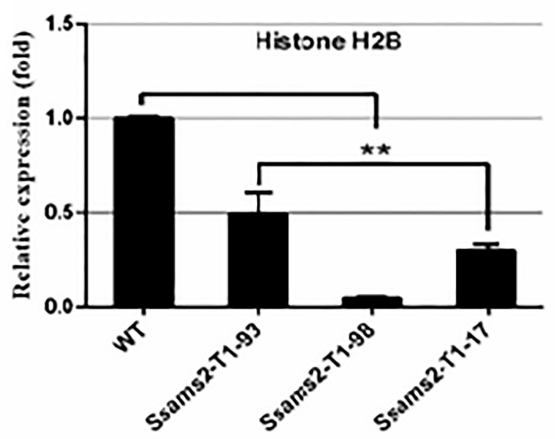

$\mathbf{F}$
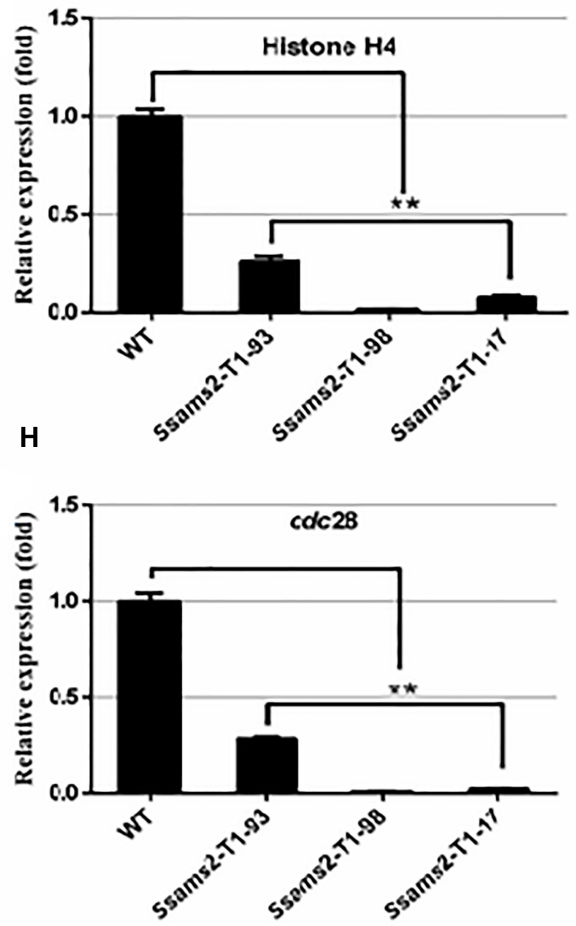

FIGURE 7 | Ssams2 is involved in the histones and cell cycle related genes transcription. (A) Function associate network of histone $\mathrm{H} 2 \mathrm{~A}$, histone $\mathrm{H} 2 \mathrm{~B}$ gene, histone $\mathrm{H} 3$ gene, histone H4 gene, CENP-A, CDC6, CDC28 in S. sclerotiorum was predicted by STRING database. The known interactions lines in pieces of in purple and in cyan, the predicted interactions lines in red, blue, and green, others lines are in yellow, black, and lilac. (B-H) $\mathrm{Histone} \mathrm{H} 2 \mathrm{~A}$ gene, histone $\mathrm{H} 2 \mathrm{~B}$ gene, histone $\mathrm{H} 3$ gene, histone $\mathrm{H} 4$ gene, CENP-A homolog gene cnp1, cdc6, and cdc28 relative expression were monitored using real-time PCR (one-way ANOVA, **indicates significance at $p<0.01)$. 
and chromosome segregation. The Ssams2-silenced stains were also slowed hyphal growth, defective in compound appressorium formation, impaired OA secreted, and reduced virulence on host plants.

\section{MATERIALS AND METHODS}

\section{Fungal Strains and Culture Conditions and Plant Materials}

The wild type isolate S. sclerotiorum "UF1" isolated from diseased petunia in Florida (Li et al., 2018) was used in this study. Strains were cultured, unless otherwise stated, on PDA medium at $25^{\circ} \mathrm{C}$ (Fan et al., 2017). S. sclerotiorum transformants were cultured on PDA amended with $100 \mu \mathrm{g} / \mathrm{ml}$ geneticin (Roche, Indianapolis, IN, United States) (Liu et al., 2018) and $50 \mu \mathrm{g} / \mathrm{ml}$ bromophenol blue (Sigma-Aldrich, United States) (Li et al., 2018). Liquid YPSU medium (50 ml containing yeast extract $4 \mathrm{~g}, \mathrm{~K}_{2} \mathrm{HPO}_{4} 1 \mathrm{~g}$, $\mathrm{Mg}_{2} \mathrm{SO}_{4} \cdot 7 \mathrm{H}_{2} \mathrm{O} 0.5 \mathrm{~g}$, sucrose $15 \mathrm{~g}, \mathrm{pH} 6.5$ ) was used to measure $\mathrm{pH}$ (Rollins, 2003). Common bean (Phaseolus vulgaris) used for pathogenicity assay grew in the greenhouse.

\section{Bioinformatics Analysis of SsAMS2}

In the S. sclerotiorum genome, nine GATA-box-containing proteins were found based on PFAM analysis (Li et al., 2017; Liu et al., 2018) and a protein (SS1G_03252) was choosed for this study. Using the MEGA software to constructed the phylogenetic tree (Liu et al., 2018). All sequences available for Ssams2 homologs form B. cinerea, M. coronariae, M. brunneum, M. majus, C. fioriniae, A. flavus, A. bombycis, T. marneffei and S. pombe. SsAMS2 (XP_001595163.1), D. rerio GATA4 (NP_571311.2), M. musculus GATA-4 (NP_001297539.1), $H$. sapiens GATA-4 (NP_001295022.1), D. melanogaster (NP_001262620.1), and S. pombe AMS2 (NP_588400.2) was performed using BlastSMART service (Liu et al., 2018).

\section{Generation of RNAi Constructs and Transformation}

The Ssams2 gene RNA-silencing vector construction strategy was described previously (Nguyen et al., 2008; Fan et al., 2017; Liu et al., 2018). The Target 1 (254 bp) and Target 2 (352 bp) fragments with HindIII restriction enzyme site were amplified with the primers pSD1-T1-F/R and pSD1-T2F/R (Supplementary Table S1). The successfully constructed plasmids used for PEG-mediated protoplasts transformation (Rollins, 2003). Transformants were cultured and purified as described previously (Fan et al., 2017). Morphological characteristics analysis and hyphal growth rate observation. Sclerotia culture and collection, colony diameters, hyphal morphology of each strain were measured or observed as described previously (Liu et al., 2018).

\section{Virulence Assays}

Seven-weeks-old common bean leaves were used as described previously (Rollins, 2003) and inoculated the mycelium plug (5 $\mathrm{mm}$ diameter) and incubated in proper environment as described previously (Qu et al., 2014; Wang et al., 2016). For the wounded treatment, a cut was made through the adaxial and abaxial surface of each leaves with a needle before inoculation. The photographs were taken with a Sony ILCE 6000. The lesion areas were calculated by tracing the necrotic area (Li et al., 2012). The experiments were performed at least three times.

\section{The Analysis of Gene Transcription Accumulation by qRT-PCR}

The genes expression was analyzed by qRT-PCR. Total RNA was isolated using TRIzol reagent according to the manufacturer's instructions (Invitrogen, Carlsbad, CA, United States) (Fan et al., 2017) and the specific methods refer to previous studies (Schmittgen et al., 2000; Gutierrez et al., 2008; Liu et al., 2018).

\section{Hyphae Cell Nuclei Numbers Assay}

Hyphae-plugs of WT strain and Ssams2-silenced strains were inoculated on PDA overlaid with cellophane $16 \mathrm{~h}$. The hyphae were stained in 4',6-diamidino-2-phenylindole (DAPI) in the dark for 30 min (Takayama et al., 2008). Protoplasts of Ssams2silenced transformants and wild type strain were prepared (Rollins, 2003) and incubated onto cellophane-overlaid PDA plates, the germinating hyphae were stained by DAPI. The same length of hyphae cell nuclei was observed and counted in the fluorescence microscope (Nikon ECLIPSE Ts2R).

\section{AUTHOR CONTRIBUTIONS}

LL, QW, and HP planned and designed the research. LL and HP wrote the manuscript. All authors performed the experiments and analyzed the data.

\section{FUNDING}

This study was financially supported by the National Key Research and Development Program of China (2018YFD0201005) and the National Natural Science Foundation of China (31772108 and 31471730).

\section{SUPPLEMENTARY MATERIAL}

The Supplementary Material for this article can be found online at: https://www.frontiersin.org/articles/10.3389/fmicb. 2018.03031/full\#supplementary-material

FIGURE S1 | Expression patterns of Ssams2. Relative expression of Ssams2 at different developmental stages (hyphae, sclerotia, and apothecium). The abundance of cDNA from hyphae was assigned a value of 1 . Gene expression values are normalized to that of the actin transcripts. Values are the means $\pm S D$ $(n=3)$. Asterisks indicate significant differences compared with the expression of Ssams2 in hyphae ${ }^{* *} P<0.01$, one-way ANOVA).

FIGURE S2 | Sequence analysis of Ssams2 in S. sclerotiorum. BLASTN search was used to show low nucleotide sequence similarities between Ssams2 and others genes in S. sclerotiorum genome.

FIGURE S3 | Identification of geneticin resistance genes by PCR. Conventional PCR were performed for geneticin resistance genes and produced $650 \mathrm{bp} \mathrm{PCR}$ products from RNAi strains. M, DNA molecular size marker; WT, wild type strain; 
lanes 1-3: putative transformants Ssams2-T1-93, Ssams2-T1-98, and Ssams2-T1-17; lanes 4-6: putative transformants Ssams2-T2-202, Ssams2-T2-102, and Ssams2-T2-108.

FIGURE S4 | Effect of Ssams2 silencing on sclerotium development in culture. (A) Comparison of sclerotia morphology and size among the WT and the silenced transformants. (B) Average sclerotium dry weight of the WT strains and the RNA-silenced transformants. (C) Average sclerotium numbers of the WT and the Ssams2 silencing strains. WT and selected RNAi-silenced transformants were

\section{REFERENCES}

Amselem, J., Cuomo, C. A., van Kan, J. A. L., Viaud, M., Benito, E. P., Couloux, A., et al. (2011). Genomic analysis of the necrotrophic fungal pathogens Sclerotinia sclerotiorum and Botrytis cinerea. PLoS Genetics 7:e1002230. doi: 10.1371/ journal.pgen.1002230

Andrew, M., Barua, R., Short, S. M., and Kohn, L. M. (2012). Evidence for a common toolbox based on necrotrophy in a fungal lineage spanning necrotrophs, biotrophs, endophytes, host generalists and specialists. PLoS One 7:e29943. doi: 10.1371/journal.pone.0029943

Boenisch, M. J., and Schäfer, W. (2011). Fusarium graminearum forms mycotoxin producing infection structures on wheat. BMC Plant Biol. 11:110. doi: 10.1186/ 1471-2229-11-110

Boland, G. J., and Hall, R. (1994). Index of plant hosts of Sclerotinia sclerotiorum. Can. J. Plant Pathol. 16, 93-108. doi: 10.1080/07060669409500766

Bolton, M. D., Thomma, B. P., and Nelson, B. D. (2006). Sclerotinia sclerotiorum (lib.) de bary: biology and molecular traits of a cosmopolitan pathogen. Mol. Plant Pathol. 7, 1-16. doi: 10.1111/j.1364-3703.2005.00316.x

Cessna, S. G., Sears, V. E., Dickman, M. B., and Low, P. S. (2000). Oxalic acid, a pathogenicity factor for Sclerotinia sclerotiorum, suppresses the oxidative burst of the host plant. Plant Cell 12, 2191-2200. doi: 10.2307/3871114

Chang, F., Riera, A., Evrin, C., Sun, J., Li, H., Speck, C., et al. (2015). CDC6 atpase activity disengages CDC6 from the pre-replicative complex to promote DNA replication. eLife 4:05795. doi: 10.7554/eLife.05795

Charron, F., Paradis, P., Bronchain, O., Nemer, G., and Nemer, M. (1999). Cooperative interaction between GATA-4 and GATA- 6 regulates myocardial gene expression. Mol. Cell. Biol. 19, 4355-4365. doi: 10.1128/MCB.19.6. 4355

Chen, E. S., Saitoh, S., Yanagida, M., and Takahashi, K. (2003). A cell cycle-regulated gata factor promotes centromeric localization of CENP-A in fission yeast. Mol. Cell. 11, 175-187. doi: 10.1016/S1097-2765(03)00011-X

Davidson, A. L., Blahut-Beatty, L., Itaya, A., Zhang, Y., Zheng, S., and Simmonds, D. (2016). Histopathology of Sclerotinia sclerotiorum infection and oxalic acid function in susceptible and resistant soybean. Plant Pathol. 65, 878-887. doi: 10.1111/ppa.12514

Fan, H., Yu, G., Liu, Y., Zhang, X., Liu, J., Zhang, Y., et al. (2017). An atypical forkhead-containing transcription factor ssfkh1 is involved in sclerotial formation and is essential for pathogenicity in Sclerotinia sclerotiorum. Mol. Plant Pathol. 18, 963-975. doi: 10.1111/mpp.12453

Franciosi, F., Tessaro, I., Dalbies-Tran, R., Douet, C., Reigner, F., Deleuze, S., et al. (2017). Analysis of chromosome segregation, histone acetylation, and spindle morphology in horse oocytes. J. Vis. Exp. 123:e55242. doi: 10.3791/55242

Gunjan, A., Paik, J., and Verreault, A. (2005). Regulation of histone synthesis and nucleosome assembly. Biochimie 87, 625-635. doi: 10.1016/j.biochi.2005.02.008

Gutierrez, L., Mauriat, M., Guenin, S., Pelloux, J., Lefebvre, J. F., Louvet, R., et al. (2008). The lack of a systematic validation of reference genes: a serious pitfall undervalued in reverse transcription-polymerase chain reaction (RTPCR) analysis in plants. Plant Biotechnol. J. 6, 609-618. doi: 10.1111/j.14677652.2008.00346.x

Guyon, K., Balague, C., Roby, D., and Raffaele, S. (2014). Secretome analysis reveals effector candidates associated with broad host range necrotrophy in the fungal plant pathogen Sclerotinia sclerotiorum. BMC Genomics 15:336. doi: 10.1186/ 1471-2164-15-336

Han, M., Chang, M., Kim, U. J., and Grunstein, M. (1987). Histone H2B repression causes cell-cycle-specific arrest in yeast: effects on chromosomal segregation, replication, and transcription. Cell 48, 589-597. doi: 10.1016/0092-8674(87) 90237-6 cultured on $9 \mathrm{~cm}$ PDA culture plates at $25^{\circ} \mathrm{C}$. Each data point represents the means \pm SD from three biological repeats $(n=3)$.

FIGURE S5 | The $\mathrm{pH}$ was measured by accurate range $\mathrm{pH}$ test paper. WT strains and the RNA-silenced transformants were inoculated in YPSU culture and incubate at $25^{\circ} \mathrm{C}$ for $24 \mathrm{~h}$ (WT) and $48 \mathrm{~h}$ (RNAi), make the clones have the same length of diameter.

TABLE S1 | Primers used in this study.

Heller, A., and Witt-Geiges, T. (2013). Oxalic acid has an additional, detoxifying function in Sclerotinia sclerotiorum pathogenesis. PLoS One 8:e72292. doi: 10. 1371/journal.pone.0072292

Hofman, T., and Jongebloed, P. (1988). Infection process of Rhizoctonia solani on Solanum tuberosum and effects of granular nematicides. Neth. J. Plant Pathol. 94, 243-252. doi: 10.1007/BF01977314

Hsia, C. W., Ho, M. Y., Shui, H. A., Tsai, C. B., and Tseng, M. J. (2015). Analysis of dermal papilla cell interactome using string database to profile the ex vivo hair growth inhibition effect of a vinca alkaloid drug, colchicine. Int. J. Mol. Sci. 16, 3579-3598. doi: 10.3390/ijms16023579

Huang, L., Buchenauer, H., Han, Q., Zhang, X., and Kang, Z. (2008). Ultrastructural and cytochemical studies on the infection process of Sclerotinia sclerotiorum in oilseed rape. J. Plant Dis. Protect. 115, 9-16. doi: 10.1007/BF03356233

Jamaux, I., Gelie, B., and Lamarque, C. (2007). Early stages of infection of rapeseed petals and leaves by Sclerotinia sclerotiorum revealed by scanning electron microscopy. Plant Pathol. 44, 22-30. doi: 10.1111/j.1365-3059.1995.tb02712.x

Jurick Ii, W. M., Dickman, M. B., and Rollins, J. A. (2004). Characterization and functional analysis of a camp-dependent protein kinase a catalytic subunit gene (pka1) in Sclerotinia sclerotiorum. Physiol. Mol. Plant Pathol. 64, 155-163. doi: 10.1016/j.pmpp.2004.07.004

Kabbage, M., Yarden, O., and Dickman, M. B. (2015). Pathogenic attributes of Sclerotinia sclerotiorum: switching from a biotrophic to necrotrophic lifestyle. Plant Sci. 233, 53-60. doi: 10.1016/j.plantsci.2014.12.018

Kim, H. J., Chen, C., Kabbage, M., and Dickman, M. B. (2011). Identification and characterization of Sclerotinia sclerotiorum nadph oxidases. Appl. Environ. Microbiol. 77, 7721-7729. doi: 10.1128/AEM.05472-11

Lecompte, F., Abro, M. A., and Nicot, P. C. (2013). Can plant sugars mediate the effect of nitrogen fertilization on lettuce susceptibility to two necrotrophic pathogens: Botrytis cinerea and Sclerotinia sclerotiorum? Plant Soil 369, 387401. doi: 10.1007/s11104-012-1577-9

Li, J., Mu, W., Veluchamy, S., Liu, Y., Zhang, Y., Pan, H., et al. (2017). The GATA-type IVb zinc-finger transcription factor Ssnsd1 regulates asexual-sexual development and appressoria formation in Sclerotinia sclerotiorum. Mol. Plant Pathol. 19, 1679-1689. doi: 10.1111/mpp.12651

Li, J., Zhang, Y., Zhang, Y., Yu, P. L., Pan, H., and Rollins, J. A. (2018). Introduction of large sequence inserts by crispr-cas 9 to create pathogenicity mutants in the multinucleate filamentous pathogen Sclerotinia sclerotiorum. mBio 9:e00567-18. doi: 10.1128/mBio.00567-18

Li, M. Y., Liang, X. F., and Rollins, J. A. (2012). Sclerotinia sclerotiorum gamma-glutamyl transpeptidase (Ss-ggt1) is required for regulating glutathione accumulation and development of sclerotia and compound appressoria. Mol. Plant Microbe Interact. 25, 412-420. doi: 10.1094/Mpmi-06-11-0159

Liang, X., Liberti, D., Li, M., Kim, Y. T., Hutchens, A., Wilson, R., et al. (2015a). Oxaloacetate acetylhydrolase gene mutants of Sclerotinia sclerotiorum do not accumulate oxalic acid, but do produce limited lesions on host plants. Mol. Plant Pathol. 16, 559-571. doi: 10.1111/mpp.12211

Liang, X., Moomaw, E. W., and Rollins, J. A. (2015b). Fungal oxalate decarboxylase activity contributes to Sclerotinia sclerotiorum early infection by affecting both compound appressoria development and function. Mol. Plant Pathol. 16, 825836. doi: 10.1111/mpp.12239

Liang, X., and Rollins, J. A. (2018). Mechanisms of broad host range necrotrophic pathogenesis in Sclerotinia sclerotiorum. Phytopathology 108, 1128-1140. doi: 10.1094/PHYTO-06-18-0197-RVW

Lim, K. K., Ong, T. Y., Tan, Y. R., Yang, E. G., Ren, B., Seah, K., et al. (2015). Mutation of histone $\mathrm{H} 3$ serine 86 disrupts gata factor ams2 expression and precise chromosome segregation in fission yeast. Sci. Rep. 5:14064. doi: 10.1038/ srep 14064 
Liu, L., Wang, Q., Sun, Y., Zhang, Y., Zhang, X., Liu, J., et al. (2018). Sssfh1, a gene encoding a putative component of the rsc chromatin remodeling complex, is involved in hyphal growth, reactive oxygen species accumulation, and pathogenicity in Sclerotinia sclerotiorum. Front. Microbiol. 9:1828. doi: $10.3389 /$ fmicb. 2018.01828

Lowry, J. A., and Atchley, W. R. (2000). Molecular evolution of the gata family of transcription factors: conservation within the DNA-binding domain. J. Mol. Evol. 50, 103-115. doi: 10.1007/s002399910012

Lumsden, R. D., and Dow, R. L. (1973). Histopathology of Sclerotinia sclerotiorum infection of bean. Phytopathology 63:708. doi: 10.1094/Phyto63-708

Lyu, X., Shen, C., Fu, Y., Xie, J., Jiang, D., Li, G., et al. (2015). The microbial opsin homolog sop1 is involved in Sclerotinia sclerotiorum development and environmental stress response. Front. Microbiol. 6:1504. doi: 10.3389/fmicb. 2015.01504

Lyu, X., Shen, C., Fu, Y., Xie, J., Jiang, D., Li, G., et al. (2016). A small secreted virulence-related protein is essential for the necrotrophic interactions of Sclerotinia sclerotiorum with its host plants. PLoS Pathog. 12:e1005435. doi: 10.1371/journal.ppat.1005435

Maruyama, T., Nakamura, T., Hayashi, T., and Yanagida, M. (2006). Histone H2B mutations in inner region affect ubiquitination, centromere function, silencing and chromosome segregation. EMBO J. 25, 2420-2431. doi: 10.1038/sj.emboj. 7601110

Marzluff, W. F. (2010). Terminating histone synthesis to preserve centromere integrity. Dev. Cell 18, 335-336. doi: 10.1016/j.devcel.2010.03.004

Nelson, D. M., Ye, X., Hall, C., Santos, H., Ma, T., Kao, G., et al. (2002). Coupling of DNA synthesis and histone synthesis in S phase independent of cyclin/cdk2 activity. Mol. Cell. Biol. 22, 7459-7472. doi: 10.1128/MCB.22.21.7459-7472. 2002

Nguyen, Q. B., Kadotani, N., Kasahara, S., Tosa, Y., Mayama, S., and Nakayashiki, H. (2008). Systematic functional analysis of calcium-signalling proteins in the genome of the rice-blast fungus, Magnaporthe oryzae, using a high-throughput RNA-silencing system. Mol. Microbiol. 68, 1348-1365. doi: 10.1111/j.1365-2958.2008.06242.x

Osley, M. A. (1991). The regulation of histone synthesis in the cell cycle. Annu. Rev. Biochem. 60, 827-861. doi: 10.1146/annurev.bi.60.070191.004143

Perlman, H., Suzuki, E., Simonson, M., Smith, R. C., and Walsh, K. (1998). GATA6 induces p21(cip1) expression and G1 cell cycle arrest. J. Biol. Chem. 273, 13713-13718. doi: 10.1074/jbc.273.22.13713

Qu, X., Yu, B., Liu, J., Zhang, X., Li, G., Zhang, D., et al. (2014). MADS-box transcription factor Ssmads is involved in regulating growth and virulence in Sclerotinia sclerotiorum. Int. J. Mol. Sci. 15, 8049-8062. doi: 10.3390/ ijms15058049

Reyes, J. C., Muro-Pastor, M. I., and Florencio, F. J. (2004). The GATA family of transcription factors in Arabidopsis and rice. Plant Physiol. 134, 1718-1732. doi: 10.1104/pp.103.037788

Rollins, J. A. (2003). The Sclerotinia sclerotiorum pac1 gene is required for sclerotial development and virulence. Mol. Plant Microbe Interact. 16, 785-795. doi: 10.1094/Mpmi.2003.16.9.785

Rollins, J. A., and Dickman, M. B. (2001). pH signaling in Sclerotinia sclerotiorum: identification of a pacC/rim1 homolog. Appl. Environ. Microbiol. 67, 75-81. doi: 10.1128/aem.67.1.75-81.2001

Schmittgen, T. D., Zakrajsek, B. A., Mills, A. G., Gorn, V., Singer, M. J., and Reed, M. W. (2000). Quantitative reverse transcription-polymerase chain reaction to study mRNA decay: comparison of endpoint and real-time methods. Anal. Biochem. 285, 194-204. doi: 10.1006/abio.2000.4753

Steadman, J. (1979). Control of plant diseases caused by sclerotinia species. Phytopathology 69:904. doi: 10.1094/Phyto-69-904

Takahashi, K., Takayama, Y., Masuda, F., Kobayashi, Y., and Saitoh, S. (2005). Two distinct pathways responsible for the loading of CENP-A to centromeres in the fission yeast cell cycle. Philos. Trans. R. Soc. Lond. B Biol. Sci. 360, 595-606; discussion 606-597. doi: 10.1098/rstb.2004.1614
Takayama, Y., Mamnun, Y. M., Trickey, M., Dhut, S., Masuda, F., Yamano, H., et al. (2010). Hsk1- and scf (pof3)-dependent proteolysis of S. pombe ams2 ensures histone homeostasis and centromere function. Dev. Cell 18, 385-396. doi: 10.1016/j.devcel.2009.12.024

Takayama, Y., Sato, H., Saitoh, S., Ogiyama, Y., Masuda, F., and Takahashi, K. (2008). Biphasic incorporation of centromeric histone CENP-A in fission yeast. Mol. Biol. Cell 19, 682-690. doi: 10.1091/mbc.e07-05-0504

Takayama, Y., Shirai, M., and Masuda, F. (2016). Characterisation of functional domains in fission yeast ams2 that are required for core histone gene transcription. Sci. Rep. 6:38111. doi: 10.1038/srep38111

Takayama, Y., and Takahashi, K. (2007). Differential regulation of repeated histone genes during the fission yeast cell cycle. Nucleic Acids Res. 35, 3223-3237. doi: $10.1093 / \mathrm{nar} / \mathrm{gkm} 213$

Tariq, V. N., and Jeffries, P. (1984). Appressorium formation by Sclerotinia sclerotiorum: scanning electron microscopy. Trans. Br. Mycol. Soc. 82, 645-651. doi: 10.1016/S0007-1536(84)80105-9

Trickey, M., Fujimitsu, K., and Yamano, H. (2013). Anaphase-promoting complex/cyclosome-mediated proteolysis of ams2 in the G1 phase ensures the coupling of histone gene expression to DNA replication in fission yeast. J. Biol. Chem. 288, 928-937. doi: 10.1074/jbc.M112.410241

Tsang, A. P., Visvader, J. E., Turner, C. A., Fujiwara, Y., Yu, C., Weiss, M., et al. (1997). Fog, a multitype zinc finger protein, acts as a cofactor for transcription factor GATA-1 in erythroid and megakaryocytic differentiation. Cell 90, 109119. doi: 10.1016/S0092-8674(00)80318-9

Uloth, M. B., Clode, P. L., You, M. P., and Barbetti, M. J. (2016). Attack modes and defence reactions in pathosystems involving Sclerotinia sclerotiorum, Brassica carinata, B. juncea and B. napus. Ann. Bot. 117, 79-95. doi: 10.1093/aob/mcv150

Valente, L. P., Dehe, P. M., Klutstein, M., Aligianni, S., Watt, S., Bahler, J., et al. (2013). Myb-domain protein Teb1 controls histone levels and centromere assembly in fission yeast. EMBO J. 32, 450-460. doi: 10.1038/emboj.2012.339

Wang, L., Liu, Y., Liu, J., Zhang, Y., Zhang, X., and Pan, H. (2016). The Sclerotinia sclerotiorum FoxE2 gene is required for apothecial development. Phytopathology 106, 484-490. doi: 10.1094/PHYTO-08-15-0181-R

Xiao, X., Xie, J., Cheng, J., Li, G., Yi, X., Jiang, D., et al. (2014). Novel secretory protein Ss-cafl of the plant-pathogenic fungus Sclerotinia sclerotiorum is required for host penetration and normal sclerotial development. Mol. Plant Microbe Interact. 27, 40-55. doi: 10.1094/MPMI-05-13-0145-R

$\mathrm{Xu}$, L., Xiang, M., White, D., and Chen, W. (2015). pH dependency of sclerotial development and pathogenicity revealed by using genetically defined oxalateminus mutants of Sclerotinia sclerotiorum. Environ. Microbiol. 17, 2896-2909. doi: 10.1111/1462-2920.12818

Yang, G., Tang, L., Gong, Y., Xie, J., Fu, Y., Jiang, D., et al. (2018). A cerato-platanin protein Sscp1 targets plant pr1 and contributes to virulence of Sclerotinia sclerotiorum. New Phytol. 217, 739-755. doi: 10.1111/nph.14842

Yu, Y., Xiao, J., Zhu, W., Yang, Y., Mei, J., Bi, C., et al. (2017). Ss-rhs1, a secretory RHS repeat-containing protein, is required for the virulence of Sclerotinia sclerotiorum. Mol. Plant Pathol. 18, 1052-1061. doi: 10.1111/mpp.12459

Zhou, W., Zhu, Y., Dong, A., and Shen, W. H. (2015). Histone H2A/H2B chaperones: from molecules to chromatin-based functions in plant growth and development. Plant J. 83, 78-95. doi: 10.1111/tpj.12830

Conflict of Interest Statement: The authors declare that the research was conducted in the absence of any commercial or financial relationships that could be construed as a potential conflict of interest.

Copyright (c) 2018 Liu, Wang, Zhang, Liu, Zhang and Pan. This is an open-access article distributed under the terms of the Creative Commons Attribution License (CC BY). The use, distribution or reproduction in other forums is permitted, provided the original author(s) and the copyright owner(s) are credited and that the original publication in this journal is cited, in accordance with accepted academic practice. No use, distribution or reproduction is permitted which does not comply with these terms. 